\title{
An Identity for Simplifying Certain Generalized Hypergeometric Functions
}

\author{
By Thomas J. Osler
}

\begin{abstract}
In this paper, an identity is proved in which certain generalized hypergeometric functions $N P^{F} N Q+N-1$ are expressed as a linear combination of $N$ functions of the $P_{Q} F_{Q}$ type. Several special cases of this identity are studied. The inverse of this identity is also given.
\end{abstract}

1. Introduction and Notation. The generalized hypergeometric function is defined by

$$
{ }_{P} F_{Q}\left(\begin{array}{l}
a_{1}, a_{2}, \ldots, a_{P} \\
b_{1}, b_{2}, \ldots, b_{Q}
\end{array} \mid z\right)=\sum_{n=0}^{\infty} \frac{\left(a_{1}\right)_{n}\left(a_{2}\right)_{n} \cdots\left(a_{P}\right)_{n} z^{n}}{\left(b_{1}\right)_{n}\left(b_{2}\right)_{n} \cdots\left(b_{Q}\right)_{n} n !},
$$

where $\left(a_{k}\right)_{n}=a_{k}\left(a_{k}+1\right)\left(a_{k}+2\right) \cdots\left(a_{k}+n-1\right)$. The series converges for all $z$ if $P<Q+1$, and for $|z|<1$ if $P=Q+1$. The series (1) is in general undefined if any of the $b_{k}$ 's are zero or a negative integer. We call $z$ the "argument" of the function, and the $a$ 's and $b$ 's are called the "parameters". To shorten the notation for the lefthand side of (1) we will sometimes write it as

$$
{ }_{P} F_{Q}\left(\begin{array}{l}
\left\{a_{j}\right\}_{j=1}^{P} \\
\left\{b_{j}\right\}_{j=1}^{Q}
\end{array} \mid z\right)={ }_{P} F_{Q}\left(\begin{array}{c}
a_{P} \\
b_{Q}
\end{array} \mid z\right) .
$$

Other devices are also used to abbreviate the notation for the generalized hypergeometric function, and in each case the meaning will be self-evident. The notations used for the special functions in this paper are those of the standard reference [4].

In [3], Lardner demonstrated relations between certain ${ }_{0} F_{3}$ functions and Bessel functions. An example is [3, (4)]

$$
{ }_{0} F_{3}(1,1 / 2,1 / 2 ; z)=1 / 2\left(J_{0}\left(4 z^{1 / 4}\right)+I_{0}\left(4 z^{1 / 4}\right)\right) \text {. }
$$

In [1], Carlson demonstrated that Lardner's relations are special cases of identities in which a certain ${ }_{2 P} F_{2 Q+1}$ function is written as a linear combination of two ${ }_{P} F_{Q}$ functions. An example is $[1,(11)]$

$$
\begin{aligned}
& 2 F_{2 Q}+1\left[\begin{array}{l}
\left\{\left\{a_{j}\right\}_{2}\right\}_{j=1}^{P} \\
\left\{\left\{b_{j}\right\}_{2}\right\}_{j=1}^{Q}, 1 / 2 \mid
\end{array} \mid z^{2}\right] \\
& \quad=\frac{1}{2}{ }_{P} F_{Q}\left[\begin{array}{l}
a_{P} \\
b_{Q}
\end{array} \mid 2^{1+Q-P_{z}}\right]+\frac{1}{2}{ }_{P} F_{Q}\left[\begin{array}{l}
a_{P} \\
b_{Q}
\end{array} \mid 2^{1+Q-P_{z}}\right],
\end{aligned}
$$

Received December 14, 1973.

AMS (MOS) subject classifications (1970). Primary 33A30; Secondary 30A10.

Key words and phrases. Hypergeometric functions, power series. 
where the notation $\{a\}_{N}$ stands for the set of $N$ parameters $a / N,(a+1) / N,(a+2) / N$, $\ldots,(a+N-1) / N$. The notation $\{a\}_{N}$ is used throughout this paper. Carlson also gives "inverse identities" in which a ${ }_{P} F_{Q}$ is expressed as a linear combination of two ${ }_{2 P} F_{2 Q+1}$ functions.

In the next section an identity (5) is derived which expands certain generalized hypergeometric functions ${ }_{N P} F_{N Q+N-1}$ as a linear combination of $N$ hypergeometric 'functions of the type ${ }_{P} F_{Q}$. The identity (5) is the principal result of this paper. An identity inverse to (5) is given by (6) in which a ${ }_{P} F_{Q}$ is expressed as a linear combination of $N$ functions of the ${ }_{N P} F_{N Q+N-1}$ type. Carlson's identities given in [1] for the generalized hypergeometric functions are the special case of (5) and (6) of this paper in which $N=2$. Carlson also gives identities for the $G$-functions which are not generalized here.

In Section 3, special cases of our general identity are examined.

In Section 4, we show that our general identity can be obtained from known formulas involving Meijer's $G$-function. A related unsolved problem is also stated.

2. The Identity and Its Inverse. Before stating and proving our identity and its inverse, we require the following lemma.

LEMmA. Let

(i) $N$ be a positive integer,

(ii) $K \in\{0,1,2, \ldots, N-1\}$,

(iii) $\omega=\exp (2 \pi i / N)$, and

(iv) $F(z)=\sum_{n=0}^{\infty} F_{n} z^{n},|z|<R$.

Then

$$
\sum_{m=0}^{\infty} F_{N m+K} z^{N m+K}=\frac{1}{N} \sum_{k=0}^{N-1} \omega^{-K k} F\left(z \omega^{k}\right) .
$$

Proof. Substituting (iv) into the right-hand side of (2) we get

$$
\begin{aligned}
\frac{1}{N} \sum_{k=0}^{N-1} \omega^{-K k} F\left(z \omega^{k}\right) & =\frac{1}{N} \sum_{k=0}^{N-1} \omega^{-K k} \sum_{n=0}^{\infty} F_{n} \omega^{k n} z^{n} \\
& =\frac{1}{N} \sum_{n=0}^{\infty}\left\{\sum_{k=0}^{N-1} \omega^{(n-K) k}\right\} F_{n} z^{n}
\end{aligned}
$$

But

$$
\begin{aligned}
\sum_{k=0}^{N-1} \omega^{(n-K) k} & =\sum_{k=0}^{N-1} \exp (2 \pi(n-K) k i / N) \\
& = \begin{cases}N & \text { if } n-K=N m, \text { for } m=0,1,2, \ldots, \\
0 & \text { otherwise. }\end{cases}
\end{aligned}
$$

Substituting (4) into (3), we get (2) at once, and the lemma is proved.

The following theorem contains the principal results of this paper.

THEOREM. Let

(i) $N$ be a positive integer, 
(ii) $K \in\{0,1,2, \ldots, N-1\}$, and

(iii) $\omega=\exp (2 \pi i / N)$.

Then

$$
\begin{aligned}
N P+1 & F_{N Q+N}\left[\begin{array}{l}
\left\{\left\{a_{j}+K\right\}_{N}\right\}_{j=1}^{P}, 1 \\
\left\{\left\{b_{j}+K\right\}_{N}\right\}_{j=1}^{Q+1}
\end{array} \mid x^{N}\right] \\
= & \frac{\Pi_{j=1}^{Q+1}\left(b_{j}\right)_{K} x^{-K} N^{(P-Q-1) K-1}}{\Pi_{j=1}^{P}\left(a_{j}\right)_{K}} \sum_{k=0}^{N-1} \omega^{-K{ }_{P} F_{Q}}\left[\begin{array}{l}
a_{P} \\
b_{Q}
\end{array} \mid \omega^{k} N^{(Q-P+1)} x\right]
\end{aligned}
$$

and

$$
\begin{aligned}
{ }_{P} F_{Q}\left[\begin{array}{l}
a_{P} \\
b_{Q}
\end{array} \mid N^{(Q-P+1)_{x}}\right]= & \sum_{K=0}^{N-1} \frac{\Pi_{j=1}^{P}\left(a_{j}\right)_{K} x^{K}}{\Pi_{j=1}^{Q+1}\left(b_{j}\right)_{K} N^{(P-Q+1) K}} \\
& \quad N P+1 F_{N Q+N}\left[\left.\begin{array}{l}
\left\{\left\{a_{j}+K\right\}_{N}\right\}_{j=1}^{P}, 1 \\
\left\{\left\{b_{j}+K\right\}_{N}\right\}_{j=1}^{Q+1}
\end{array}\right|^{N}\right],
\end{aligned}
$$

where we define $b_{Q+1}=1$. Assume also that none of the denominator parameters in the above hypergeometric functions is zero or a negative integer so that these functions are defined. If $P=Q+1$, then $|x|<1$, but if $P<Q+1$, then $x$ is unrestricted.

Remark. The ${ }_{N P+1} F_{N Q+N}$ appearing in the conclusion of the above theorem is really an ${ }_{N P} F_{N Q+N-1}$. This is due to the definition of $b_{Q+1}=1$, causing the numerator parameter " 1 " to be cancelled by one of the denominator parameters $\left(b_{Q+1}+K\right) / N,\left(b_{Q+1}+K+1\right) / N, \ldots,\left(b_{Q+1}+K+N-1\right) / N$.

Proof. Set

$$
F(z)={ }_{P} F_{Q}\left(\begin{array}{l}
a_{1}, a_{2}, \ldots, a_{P} \\
b_{1}, b_{2}, \ldots, b_{Q}
\end{array} \mid z\right)
$$

in the lemma and get

$$
\frac{1}{N} \sum_{k=0}^{N-1} \omega^{-K k}{ }_{P} F_{Q}\left(\begin{array}{c}
a_{1}, \ldots, a_{P} \\
b_{1}, \ldots, b_{Q}
\end{array} \mid \omega^{k} z\right)=\sum_{m=0}^{\infty} \frac{\prod_{j=1}^{P}\left(a_{j}\right)_{N m+K}}{\prod_{j=1}^{Q+1}\left(b_{j}\right)_{N m+K}} z^{N m+K}
$$

But $\left(a_{j}\right)_{N m+K}=\left(a_{j}\right)_{K}\left(a_{j}+K\right)_{N m}$, and from [4, p. 9, (10)] we get

$$
\left(a_{j}\right)_{N m+K}=N^{N m}\left(a_{j}\right)_{K} \prod_{k=0}^{N-1}\left(\left(a_{j}+K+k\right) / N\right)_{m}
$$

Substituting this result for $\left(a_{j}\right)_{N m+K}$ and the corresponding product for $\left(b_{j}\right)_{N m+K}$ into the right-hand side of (7), we get the right-hand side of (7) equal to

$$
\frac{z^{K} \Pi_{j=1}^{P}\left(a_{j}\right)_{K}}{\prod_{j=1}^{Q+1}\left(b_{j}\right)_{K}} \sum_{m=0}^{\infty} \frac{\Pi_{j=1}^{P} \Pi_{k=0}^{N-1}\left(\left(a_{j}+K+k\right) / N\right)_{m}}{\Pi_{j=1}^{Q+1} \Pi_{k=0}^{N-1}\left(\left(b_{j}+K+k\right) / N\right)_{m}} \frac{(1)_{m}}{m !} \frac{N^{N m P} z^{N m}}{N^{N m(Q+1)}}
$$

where we note that $(1)_{m} / m !=1$. Set $x=N^{(P-Q-1)} z$ and we obtain our result (5) at once. 
To prove the inverse identity (6), first bring the factor multiplying $\Sigma_{k=0}^{N-1}$ on the right-hand side of (5) over to the left-hand side of (5). Next, sum both sides over $K$ from 0 to $N-1$ and note that on the right-hand side $\Sigma_{K=0}^{N-1} \omega^{-K k}$ is equal to $N$ when $k=0$, but vanishes otherwise. Thus (6) is demonstrated and the proof of our theorem is complete.

Before continuing, we note that Carlson's relations for the generalized hypergeometric functions in [1] are special cases of our (5) and (6) in which $N=2$. The first of Carlson's identities (11) is the special case of our (5) in which $N=2$ and $K=0$, and the second of Carlson's (11) is the special case of our (5) in which $N=2$ and $K=1$. Carlson's (10) is the special case of our (6) in which $N=2$.

Having stated and proved our general identity (5) and its inverse (6), we proceed to study special cases in the next section.

3. Special Cases. In the following, $P, Q, N$ and $K$ are given specific numerical values in (5) so that we can observe more clearly the specific forms assumed by the identity. We assume throughout that the denominator parameters in the hypergeometric functions are never nonpositive integers.

Case 1. Take $P=0, Q=0$, and $K=0$ in (5) and get

$$
{ }_{0} F_{N-1}\left(\overline{1 / N, 2 / N, \ldots,(N-1) / N} \mid x^{N}\right)=\frac{1}{N} \sum_{k=0}^{N-1} \exp [N x \exp (2 \pi k i / N)] \text {. }
$$

Case 2. Take $P=1, Q=0, N=2, K=0$ and $a_{1}=2 a$ in (5) and get

$$
{ }_{2} F_{1}\left(\begin{array}{c}
a, a+(1 / 2) \\
1 / 2
\end{array} \mid x^{2}\right)=\frac{1}{2}\left[(1-x)^{-2 a}+(1+x)^{-2 a}\right] \text {. }
$$

This is a well-known elementary relation $[1$, p. 101, (5)]

Case 3. Take $P=1, Q=0, N=2, K=1$ and $a_{1}=2 a$ in (5) and get

$$
{ }_{2} F_{1}\left(\begin{array}{c}
a+(1 / 2), a+1 \\
3 / 2
\end{array} \mid x^{2}\right)=\frac{1}{4 a x}\left[(1-x)^{-2 a}-(1+x)^{-2 a}\right] .
$$

Case 4. Take $P=2, Q=1, N=2, K=0, a_{1}=2 a, a_{2}=2 b$ and $b_{1}=2 c$ in (5) and get

$$
\begin{aligned}
& { }_{4} F_{3}\left(\begin{array}{c}
a, a+(1 / 2), b, b+(1 / 2) \\
c, c+(1 / 2), 1 / 2
\end{array} \mid x^{2}\right) \\
& =\frac{1}{2}\left[{ }_{2} F_{1}\left(\begin{array}{c}
2 a, 2 b \\
2 c
\end{array} \mid x\right)+{ }_{2} F_{1}\left(\begin{array}{c}
2 a, 2 b \\
2 c
\end{array} \mid-x\right)\right] \text {. }
\end{aligned}
$$

Case 5. Take $P=2, Q=1, N=2, K=1, a_{1}=2 a, a_{2}=2 b$, and $b_{1}=2 c$ in (5) and get

$$
\begin{aligned}
{ }_{4} F_{3}\left(\begin{array}{c}
a+(1 / 2), a+1, b+(1 / 2), b+1 \\
c
\end{array}+(1 / 2), c+1,3 / 2\right. & \left.x^{2}\right) \\
= & \frac{c}{4 a b x}\left[{ }_{2} F_{1}\left(\begin{array}{c}
2 a, 2 b \\
2 c
\end{array} \mid x\right)-{ }_{2} F_{1}\left(\begin{array}{c}
2 a, 2 b \mid-x \\
2 c
\end{array}\right)\right] .
\end{aligned}
$$


Case 6. Take $P=2, Q=1, N=3, K=0, a_{1}=3 a, a_{2}=3 b$, and $b_{1}=3 c$ in (5) and get

$$
\begin{gathered}
{ }_{6} F_{5}\left(\begin{array}{c}
\left.a, a+(1 / 3), a+(2 / 3), b, b+(1 / 3), b+(2 / 3) \mid x^{3}\right) \\
c, c+(1 / 3), c+(2 / 3), 1 / 3,2 / 3
\end{array}\right) \\
=\frac{1}{3} \sum_{k=0}^{2}{ }_{2} F_{1}\left(\begin{array}{c}
3 a, 3 b \\
3 c
\end{array} \mid x \exp (2 \pi k i / 3)\right) .
\end{gathered}
$$

Case 7. Set $x=-1,3 a=\alpha+(1 / 3), 3 b=3 \alpha$ and $3 c=2 \alpha+(2 / 3)$ in the previous example. We can evaluate the three ${ }_{2} F_{1}$ 's of special argument using [1, pp. 104105, (47), (55), (56)] and we obtain

$$
\begin{gathered}
{ }_{6} F_{5}\left(\begin{array}{c}
(3 \alpha+1) / 9,(3 \alpha+4) / 9,(3 \alpha+7) / 9, \alpha, \alpha+(1 / 3), \alpha+(2 / 3) \mid-1) \\
(6 \alpha+2) / 9,(6 \alpha+5) / 9,(6 \alpha+8) / 9,1 / 3,2 / 3
\end{array}\right) \\
=\frac{\Gamma((6 \alpha+2) / 3)}{3}\left\{\frac{2^{-3 \alpha} \sqrt{\pi}}{\Gamma((3 \alpha+4) / 6) \Gamma((3 \alpha+1) / 2)}\right. \\
\left.+\frac{3^{-(3 \alpha+1) / 2} 4 \pi \cos (\pi \alpha / 2)}{\Gamma(2 / 3) \Gamma((3 \alpha+1) / 3) \Gamma((3 \alpha+2) / 3)}\right\} .
\end{gathered}
$$

4. Relation with Meijer's G-Function. Yudell Luke has observed that our inverse identity (6) can also be obtained from known formulas involving Meijer's $G$-function. We simply outline how this is done, since the derivation given in this paper of our inverse identity (6) and the identity (5) makes use only of the very elementary formula (2) for power series and is in this sense preferable. To obtain (6) by the use of $G$ functions, set $m=1$ in Eq. (5) of [4, vol. 1, p. 150] and then use Eqs. (7) and (12) of $[4$, vol. 1, pp. 145 and 146].

As a final remark we state an unsolved problem. Carlson gives identities for the $G$-function which resemble our identities (5) and (6) for the hypergeometric function in the special case $N=2[1$, p. 237, (20) and (21)]. In his identities, Carlson expresses one $G$-function in terms of two related $G$-functions, just as we have expressed one hypergeometric function in terms of $N$ related hypergeometric functions in (5) and (6). Can one generalize Carlson's identities so that now one $G$-function is expressed in terms of $N G$-functions? It would seem that such identities should exist, but thus far they have eluded this author.

5. Acknowledgments. This paper evolved by pursuing a remark made by Professor Richard Askey of the University of Wisconsin in which he suggested that the author study his Leibniz rule in [5] after applying Legendre's duplication formula. After examining this remark the author discovered that the same results could be obtained with greater ease from the lemma in this paper. The author wishes to thank Professor Yudell Luke of the University of Missouri-Kansas City, for calling the author's attention to the work of Carlson and Lardner. 
1. B. C. CARLSON, "Some extensions of Lardner's relations between ${ }_{0} F_{3}$ and Bessel functions," SIAM J. Math. Anal., v. 1, 1970, pp. 232-242. MR 41 \#3819.

2. A. ERDÉLYI ET AL., Higher Transcendental Functions. Vol. 1, McGraw-Hill, New York, 1953. MR 15, 419.

3. T. J. LARDNER, "Relations between ${ }_{0} F_{3}$ and Bessel functions," SIAM Rev., v. 11, 1969, pp. 69-72. MR 42 \#2048.

4. Y. L. LUKE, The Special Functions and Their Approximations. Vol. 1, Math. in Sci. and Engineering, vol. 53, Academic Press, New York, 1969. MR 39 \#3039.

5. T. J. OSLER, "A further extension of the Leibniz rule to fractional derivatives and its relation to Parseval's formula," SIAM J. Math. Anal., v. 3, 1972, pp. 1-16. MR 48 \#2323a. 